Voix et Images

\title{
Gaston Miron : le rythme, le sens, le sujet
}

\section{Chantal de Grandpré}

Volume 10, numéro 3, printemps 1985

André Major

URI : https://id.erudit.org/iderudit/200519ar

DOI : https://doi.org/10.7202/200519ar

Aller au sommaire du numéro

\section{Éditeur(s)}

Université du Québec à Montréal

ISSN

0318-9201 (imprimé)

1705-933X (numérique)

Découvrir la revue

Citer cet article

de Grandpré, C. (1985). Gaston Miron : le rythme, le sens, le sujet. Voix et

Images, 10(3), 126-136. https://doi.org/10.7202/200519ar d'utilisation que vous pouvez consulter en ligne.

https://apropos.erudit.org/fr/usagers/politique-dutilisation/ 


\title{
Gaston Miron: le rythme, le sens, le sujet 1
}

\author{
par Chantal de Grandpré, Université du Québec à Montréal
}

En juxtaposant le rythme, le sens et le sujet, cette étude tente de désamorcer l'opposition stérile entre analyse "thématique» et analyse "formelle», mettant de l'avant l'indissociabilité de ces trois éléments: le rythme est pris ici en tant qu'élément structurant du sens, lequel est luimême le produit d'un sujet. La théorisation de cette indissociabilité s'effectue principalement à partir des travaux d'Henri Meschonnic2, du travail étymologique fait sur le mot «rythme» par Émile Benveniste3 et des intuitions théoriques d'Ezra Pound qui déjà revendiquait une telle indissociabilité;

I believe in an 'absolute rythm', a rythm, that is, in poetry which corresponds exactly to the emotion or shade of emotion to be expressed. A man's rythm must be interpretative, it will be, therefore, in the end, his own, uncounterfeiting, uncounterfeitable 4 .

Une définition linguistique du rythme apparaît par conséquent comme l'enjeu principal de cette théorisation qui refuse de laisser le rythme au seul domaine de la métrique où celui-ci, identifié au mètre, est réduit à n'être qu'un élément formel, répétitif. En retirant de la sorte le rythme du discours pour le mettre dans le mètre, la métrique asémantise le texte et suppose par ailleurs que le langage parlé et la prose n'ont pas de rythme, ce qui n'est pas vrai. Cette réduction qui fait du rythme une notion définie à partir du retour régulier des temps forts, des accents et des césures, et qui repose sur une conception syllabique du vers français, procède de la fausse étymologie du mot rythme que Benveniste a critiquée, rendant par là possible une définition linguistique du rythme qui prenne celui-ci comme l'organisation spécifique des marques d'un discours. Benveniste a indiqué que l'association du rythme au mètre a été faite par Platon et qu'avant lui, chez Démocrite plus particulièrement, le rythme signifiant "forme distinctive ${ }^{5}$ » forme de ce qui est sujet à changement. La fausse étymologie qui, à partir de Platon, fait du rythme une régularité continue pourtant d'être postulée et s'inscrit par conséquent dans des stratégies. Il ne s'agit pas tant ici d'opposer une définition vraie à une ou plusieurs qui seraient fausses, mais de montrer que chaque définition est chaque fois une stratégie et que celle que j'ai adoptée pose les rapports entre sens, sujet et histoire.

Le rythme linguistique est fait d'accents d'intensité et d'accents prosodiques. Il est l'organisation de ces marques accentuelles, qu'elles soient lexicales, prosodiques ou syntaxiques, opérant tant au niveau syntagmatique qu'au niveau paradigmatique de l'ouvre et neutralisant justement par là la notion de niveau dans la mesure où le texte est système et où tous ses constituants sont inséparables. L'analyse rythmique est une analyse du mode de signifier du texte; sa technicité débouche non sur la formalisation de ses propres concepts, mais sur la recherche de l'organisation de la signi- 
fiance et de la signification du discours. Dans cette optique, le rythme remet en question la notion de sens dans son acceptation lexicale en faisant davantage appel à la notion de valeur qui se joue dans l'organisation du marqué et du non marqué.

On parle donc des formes-sens d'un texte, où ce qui est dit et la façon dont ça l'est sont inséparables: "C'est singulier en littérature, quand la forme n'est pas nouvelle, le fond ne l'est pas non plus ${ }^{7} »$. Ce concept de forme-sens s'inscrit contre le dualisme du signifiant et du signifié qu'une lecture hjelmslevienne de Saussure réactualise et que Benveniste a critiquée:

Entre le signifiant et le signifié, le lien n'est pas arbritraire; il est nécessaire. (...) Le signifiant est la traduction phonique d'un concept; le signifié est la contre-partie mentale d'un signifiant. Cette consubstantialité du signifiant et du signifié assure l'unité structurale du signe linguistique. (...) Ce qui est arbitraire, c'est que tel signe, et non tel autre, soit appliqué à tel élément de la réalité, et non à tel autre8.

L'étude du rythme des textes désamorce par conséquent le dualisme du fond et de la forme. On y voit la prosodie, être, comme le dit Henri Meschonnic «la face sonore d'une syntaxe9». Il ne s'agit pas, en ce qui concerne celle-ci, de doter certaines lettres, certains sons, d'un sens métaphysique, ou de leur trouver une base universelle à la manière de Fónágy 10 , mais bien de tenter l'exploration d'une prosodie-sens où des sons sont porteurs de sens en fonction du sens des mots dans lesquels ils se trouvent et de leur fonctionnement dans le texte, devenant en quelque sorte métaphores rythmiques.

Une des formes-sens fondamentales chez Miron est liée au mot «terre». C'est dans le recueil Courtepointes 11 surtout 'que s'instaure cette relation privilégiée avec la terre et que la chaîne prosodique/tER/ se retrouve constamment. En établissant ainsi un rapport avec la terre plutôt qu'avec le pays, comme c'est le cas dans l'Homme rapaillé12, Miron va davantage dans le sens d'une ontologie que d'un nationalisme, et dès le premier poème de Courtepointes, la constitution de son être procède de celle de la terre. Avant d'exister syntaxiquement au troisième vers de la première strophe, le sujet est introduit par ses composantes primordiales.qui sont la terre et l'eau:

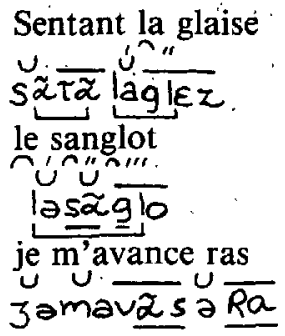




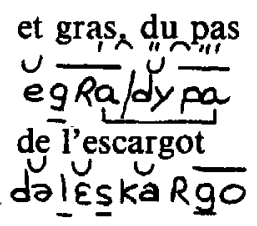

Les deux premiers vers sont étroitement mêlés prosodiquement, tout comme l'eau et la terre le sont, qui président à la constitution du «je». Le sanglot prend une importance particulière du fait qu'il constitue un vers à lui seul et qu'on y retrouve l'ensemble des éléments prosodiques du premier vers

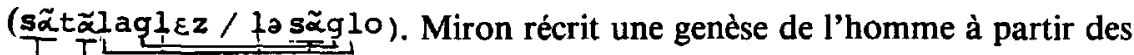
mêmes éléments qu'on trouve dans la Genèse. Il y ajoute la tristesse, puisque l'élément liquide dont il s'agit est le sanglot, tristesse dont il dira dans les Notes sur le non-poème et le poème ${ }^{13}$ qu'elle est ontologique:

Le non-poème

c'est ma tristesse

ontologique

la souffrance d'être un autre

Le sujet «je» est nommé au troisième vers et associé à l'action d'avancer. Dans ce syntagme verbal, la chaîne prosodique en/ $\tilde{\alpha} s /$ des deux premiers vers se continue, indicative de la consubstantialité existant entre la glaise, le sanglot et le sujet. Par la suite; par le biais du pas, le sujet se compare à l'escargot dont le mouvement est en rapport étroit avec la terre (je m'avance ras). Le rapprochement congénital entre la terre et l'homme est accentué de plus par la brièveté des vers où les chaînes prosodiques apparaissent collées les unes aux autres.

Cette interpénétration de la terre et du sujet, établie d'emblée par le tout premier poème du recueil, se retrouve ensuite constamment:

Cette terre dans mes épaules

cette branche qui dans ma voix bruit

(En Archambault)

terre tour à tour taciturne et tourmenteuse

terre tout à la fois en chaleur et frileuse

pour qu'un jour enfin je repose

dans ton envolée la plus basse

(Dans mes arpents d'yeux)

Elle s'actualise par l'utilisation d'adjectifs qui syntaxiquement devraient qualifier une contrée, un pays, et qui qualifient le(s) sujet(s):

tête davantage pluvieuse, ma très-très tête au loin

toi ma giboyeuse

(Rue Saint-Christophe)

(Cinquième partir) 
Ce dernier exemple illustre bien l'indissociabilité de tous les éléments d'un texte: syntaxique, prosodique, accentuel et sémantique. Miron utilise l'adjectif "giboyeuse" pour dire le lien femme/terre. La femme avait déjà été qualifiée de "gigoteuse" et de "voyageuse" dans le même poème. Il y a récurrence du son $/ z \otimes /$ pour ces trois adjectifs. Dans le dernier cas (giboyeuse), un glissement syntaxique-sémantique s'opère qui a été annoncé au moment de l'utilisation de l'adjectif "voyageuse":

$$
\begin{aligned}
& \text { voyageuse d'air léger de rêves cêréales }
\end{aligned}
$$

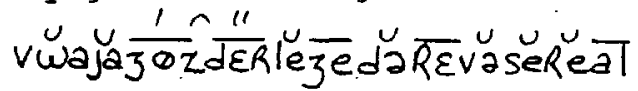

On voit le son $/ z 0 /$ de "voyageuse" directement accolé, par le biais du contre-accent rythmique, au son / $\varepsilon R /$ de "air" qui reprend partiellement la chaîne prosodique/teR/. Il y a dans ce rapprochement rythmique et prosodique un indice du rapprochement sémantique à venir et déjà inscrit en filigrane. La prosodie, les accents et la syntaxe concourent ensemble à créer l'effet de sens qui insiste sur l'interpénétration du sujet (ici la femme) et de la terre.

La chaîne prosodique / $t \varepsilon R /$ apparaît de façon partielle dans d'autrè mots du recueil, renvoyant nommément au sens du mot "terre" de façon évidente comme c'est le cas pour le mot "hiver", associé de longue date dans la symbolique québécoise à l'idée de tèrre, et fonctionnant comme tel dans le poème «En Archambault»:

Cette terre dans mes épaules

cette branche qui dans ma voix bruit

c'est déjà et encore l'hiver

Ou encore pour le mot «père» qui dans «Fragment de la vallée» est lié au travail de la terre. La chaîne \%per/ se retrouve dans «empierrée» qui qualifie la montagne:

sur tes pentes hirsutes

la courbure séculaire des hommes

contre la face empierrée des printemps montagneux

je me défais à leur encontre

de la longue lente prostration des pères

La chaîne / $t \varepsilon R /$ se retrouve aussi, intégralement cette fois, dans des mots qui donnent à la terre une dimension nouvelle, comme c'est le cas pour «diamantaire» et pour «éternité». Ce n'est donc pas par hasard si, dans les poèmes de Femme sans fin 14 , la femme devient «éternelle», elle qui comme la terre met au monde. Il y a continuité de cette thématique à travers l'ensemble de l'œuvre de Miron, mais c'est dans Courtepointes qu'elle est la plus élaborée, qu'elle fonde la problématique du recueil.

Il y a dans Courtepointes une volonté, non seulement de dire l'essentiel, mais surtout d'organiser cet essentiel, et c'est ce souci qui à première 
vue distingue Courtepointes de l'Homme rapaillé. Courtepointes est davantage un recueil de poèmes que ne l'est l'Homme rapaillé dont la vocation est plus anthologique. Ce qui frappe ensuite, c'est la brièveté des textes; à part les cinquième et sixième parties, plus longues, en continuité avec les cycles de "La marche à l'amour» et de «La batèche», la brièveté est une caractéristique dominante dans Courtepointes. Ce parti pris de brièveté met dans un rapport d'immédiateté les éléments que Miron veut rapprocher et dont l'homme et la terre sont les plus primordiaux. Mais ce rapprochement ontologique n'a de force qu'en ce qu'il est politique, qu'en ce qu'il subvertit un conception chrétienne de l'homme qui a servi à l'exploitation de cet homme. La terre est pendant près de deux siècles le lieu d'un confinement qui paradoxalement mène à la dépossession de la terre. Son historicité est lourdement marquée par la mainmise de l'Église dont ce fut «l'âge d'or 15 » et qui a établi avec la communauté canadienne-française «un pacte éternel avec la terre16». En écrivant une genèse de l'homme, Miron radicalise et politise celle-ci puisqu'il le fait dans le but de revendiquer ensuite cette terre en posant que la dépossession de celle-ci n'équivaut ni plus ni moins qu'à la dépossession congénitale de son être même.

Le rapport au pays est bien différent de celui avec la terre. Dans les poèmes de «La vie agonique», il est un rapport de tension qui fait hésiter entre la vie et la mort. La terre est résolument du côté de la vie, alors que le pays hésite. Cette tension est la plus perceptible dans le poème «Les siècles de l'hiver». où il y a couplage entre le pays et le sujet, où l'on voit à la fin le sujet investi par le pays, lui-même investi par l'hiver, mais où cette interprétation n'est pas réciproque.

Le couplage des pronoms personnels «tu» et «nous» est aussi un parallélisme par lequel chacun des deux termes tente de s'affirmer mais où, en dernier ressort, l'impuissance domine, cette impuissance qui leur est commune et qui les sépare:

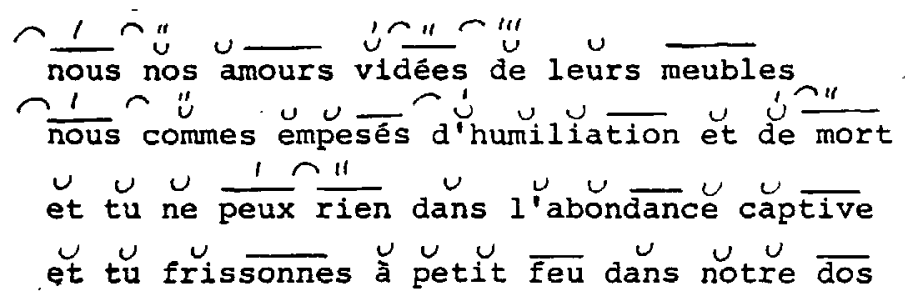

Le «nous » dépossédé est en position marquée en début de vers mais les marques de sa dépossession sont aussi accentuees; il y a chaîne prosodique en /d/ qui se retrouve dans «vidées de»(vi $\overline{d e d a) ~ e t ~ d a n s ~ « d ' h u m i l i a-~}$ tion» (dýmil jasj l'impuissance du pays auquel le sujet tourne le dos au dernier vers.

Ce jeu sur les pronoms personnels, on le retrouve aussi dans «Héritage de la tristesse» où le pays agonisant est décrit tout au long du poème par le 
pronom personnel «il», celui qui est la marque du non-sujet:

Il faut voir que la définition ordinaire des pronoms personnels comme contenant les trois termes $j e, t u, i l$, y abolit justement la notion de «personne». Celle-ci est propre seulement à $j e / t u$, et fait défaut dans $i l 17$.

Ả la fin du poème il y a couplage entre le «il» et le «nous»:

vents ameutez nous, et de vos bras de fleuve ensemble enserrez son visage de peuple abîmé, redonnez-lui la chaleur

Dans ce couplage, le temps d'un vers, il y a eu transsubstantiation, le pays est devenu "nous», mais en dernière instance, dans l'espoir d'un avenir possible, le pronom «lui» refait son apparition, rétablissant la distance entre le sujet et le pays/peuple.

Avec le pays, c'est la recherche conflictuelle d'une identité qui domine. Cet aspect est accentué du fait qu'entre le sujet de l'écriture, masculin, et le pays, lui aussi masculin, ce type de rapport peut s'établir puisqu'il y a motivation par le discours (deux masculins). Avec le pays, il y a identité en même temps que conflit; avec la terre, il y a ressemblance en même temps qu'indissociabilité.

Avec la terre, simultanément, le noir apparaît comme un mot-valeur qui lui aussi traverse l'œuvre qui est davantage marqué dans Courtepointes. Or, les poèmes de Courtepointes ont pour la plupart été écrits pendant la décennie des années cinquante, celle de la "grande noirceur», et surtout, Miron les a datés, insistant de ce fait sur le moment de leur écriture.

Le noir investit les poèmes de Courtepointes; il noircit même le blanc qui se rattache à l'hiver qui qualifie toute la période historique d'avant le dégel des années soixante:

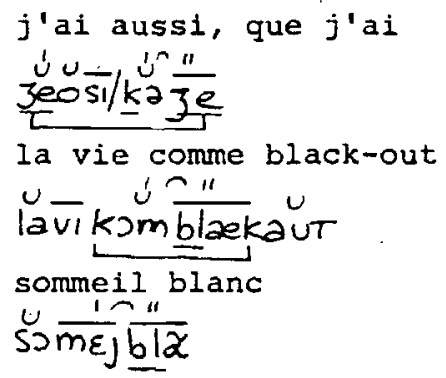

L'utilisation répétée du verbe "avoir» insiste ici sur ce que le sujet possède véritablement, une vie qui est néantisée. La ressemblance qui unit le noir au blanc est rendue manifeste par l'anglicisation du noir, anglicisation qui est un des facteurs essentiels de l'aliénation; le noir et le blanc ont en commun 
le son /bl/ qui les fait se ressembler. Si «black» est en position accentuée, c'est que l'usage québécois privilégie une prononciation anglaise des termes anglais au contraire des Français qui eux, accentueraient, selon l'usage français, la dernière syllabe, soit le «out». «Black-out» est ici un pied dactyle; la métrique anglaise est intacte. Dans l'usage québécois, il s'agit d'ailleurs là d'un problème de fond plutôt que d'une simple querelle de diction. C'est un problème qui touche à la situation paradoxale dans laquelle l'anglais est tenu. D'une part, il s'agit d'une langue haïe, et sa prédominance est une menace constante dont on peut prendre la mesure en examinant le travail de déstructuration du français déjà accompli. Mais en même temps, c'est une langue prestigieuse à laquelle on n'ose pas, par exemple, imposer le rythme et la prononciation du français lorsqu'on lui emprunte des mots.

Le phénomène de noircissure du blanc se retrouve dans un autre poème, «Le Vieil Ossian», quand Miron écrit:

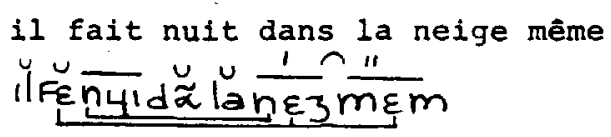

La prosodie et les accents rythmiques font ici se ressembler la nuit, noire, et la neige, insistant sur la noirceur de la neige dans la mesure où le mot est en position de contre-accent rythmique.

Le noir est, comme on l'a dit, politique; il est clairement associé à l'analphabétisme:

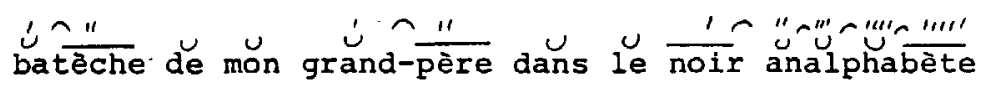

(La Batèche)

Ả la misère morale et sociale:

$$
\text { sa nuit de merveille et de misère } \frac{u}{\text { noire dans }} \cup \text { le vent }
$$

(En Archambault)

Toujours en position accentuée, on le retrouve dans (La vie agonique):

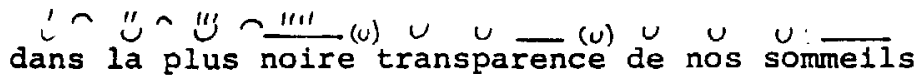

$$
\begin{aligned}
& \text { (L'homme agonique) }
\end{aligned}
$$

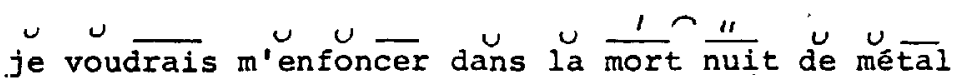

$$
\begin{aligned}
& \text { (Monologues de l'aliénation délirante) }
\end{aligned}
$$

Et il est jusque dans les poèmes de Femme sans fin: 


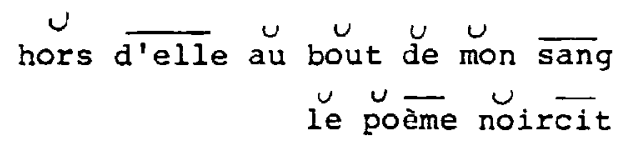

(Passages de l'amnésie)

Ce qui s'oppose au noir, ce n'est pas le blanc mais la clarté. Voilà pourquoi, dans Courtepointes où le noir fonctionne nẻgativement, Miron rendra la terre claire, elle qui logiquement devrait être noire:

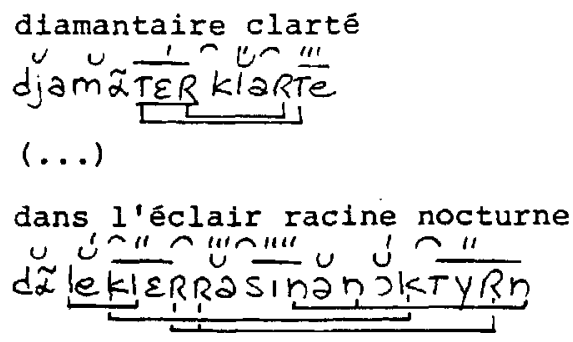

(Fragment de la vallée)

La terre, inscrite ici dans le mot «diamantaire» par le biais de la chaîne prosodique / $t \varepsilon R /$ est en position de contre-accent rythmique-prosodique avec "clarté». Dans le deuxième vers cité, il y a encore contre-accent rythmiqueprosodique qui porte sur «éclair» et "racine», le deuxième terme renvoyant indirectement à la terre. fin:

Cette clarté rejaillira d'ailleurs sur le noir des poèmes de Femme sans

par la vaste noirceur éblouissante

(Ce désir...)

Après s'être réapproprié la terre, c'est le noir que Miron se réapproprie, assumant le noir politique pour mieux le transformer, l'éclairer. Dans une poétique qui ne cesse de dire que «l'avenir est aux sources» et que le présent est fait du passé, les éléments qui sont les signes de l'oppression sont transformés, retournant celle-ci contre elle-même.

Le noir clair est déjà en filigrane dans Courtepointes, dans «Fragment de la vallée» où l'oiseau, une corneille, noire, est l'indice d'une liberté possible:

dans l'éclair racine nocturne

le firmament se cabre et de crête en crête

va la corneille au vol balourd

émouvante voix de balise

Cette corneille, on la retrouve dans "La vie agonique":

Corneille, ma noire

corneille qui me saoûle 
opaque et envoûtante

venue pour posséder ta saison et ta descendance

déjà l'été goûte un soleil de mûres

déjà tu conjoins en ton vol la terre et l'espace

Miron dit "Corneille, ma noire» - le noir qu'on possède n'est pas le noir qu'on subit. C'est un noir transformé, ici encore, éclairé par un «soleil de mûres».

Le non-dualisme du fond et de la forme chez Miron se manifeste également par l'incursion de l'oral dans l'écrit. C'est sans doute dans «La batèche" que le rythme de la langue parlée investit le plus l'écriture des poèmes. Dans ce cycle, Miron utilise la manifestation linguistique la plus émotive et la plus caractéristique de la langue parlée, le juron. Dans sa spécificité québécoise, le juron met en cause l'Église dans sa présence la plus quotidienne; c'est contre elle et les élites qu'elle a formées que Miron reconstitue le juron et en fait une strophe:

Sainte Bénite de Sainte Bénite de batèche

Sainte Bénite de vie magannée

belle grégousse de vieille réguine de batèche

Et qu'il historicise sa révolte dans un vers qui reprend la structure syntaxique d'une expression-cliché:

Moi le raqué de partout batèche

nous les raqués de l'histoire batèche

C'est la langue de "parlures» et de «sacrures», la langue de la majorité analphabétisée qu'il revendique contre la langue des «insectes des belles manières", comme il nomme les élites.

En choisissant le juron, c'est un certain type de tradition orale qu'il choisit, celle qui exprime la révolte, celle qui a permis que la langue française continue d'être parlée. Ce n'est pas un hasard si Miron mentionne le «noir analphabète» justement dans «La batèche»; c'est qu'il est conscient que l'Église, loin d'avoir conservé quoi que ce soit, a surtout travaillé à analphabétiser une population qu'elle pouvait ainsi beaucoup mieux diriger. C'est cette même population qui, par contrecoup, en continuant de parler français, $a$ instauré une tradition orale dans laquelle s'inscrivent une continuité et une historicité.

En se situant de la sorte du côté de l'oralité, Miron pose un choix linguistique qui est un choix historique, car toute une conception de l'homme est en jeu dans ce choix, le mépris du langage ordinaire coïncidant la plupart du temps avec le mépris de l'homme ordinaire. 
Les poèmes d'amour, surtout «La marche à l'amour», sont aussi du côté de l'oral. Cela dit, la prédilection de Miron pour l'oral ne s'oppose pas à l'écrit, elle l'investit, ce qui est différent:

Le texte-lecture est un acte individuel-collectif, un passage trans-énonciatif d'un signifiant, le texte, à un autre signifiant, le lecteur. Cela dans l'oral, qui n'est ni le parler opposé à l'écrit, ni le privilège de la phonie sur la graphie, mais une unité à dialectique interne de l'ouvre et du dire18.

L'histoire de l'oppression s'écrit dans les poèmes d'amour où l'histoire de l'oppression de la femme prend figure de révélateur. Mise au passé, en même temps que son passé est dit, elle devient ensuite la couseuse, celle dont les instruments deviennent des instruments de libération pour l'homme:

tu es mon amour ma ceinture fléchée d'univers ma danse carrée des quatre coins d'horizon le rouet des échevaux de mon espoir

Plus que des étoffes, c'est le tissu même du pays que tisse la femme. Rien d'étonnant à ce que le deuxième recueil de Miron s'intitule justement Courtepointes. En quoi, on voit que les poèmes d'amour s'ancrent aussi dans le politique.

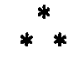

Le rôle transformateur de la poésie de Miron s'actualise dans le travail prosodique et syntaxique de son écriture:

Mais c'est parce que l'individu est ce passage, qui n'est pas seulement passage d'une histoire, qu'il peut agir sur cette histoire. Le poème, le rythme, activités de sens, sont des éléments de transformation 19 .

Miron tire de nouveau l'homme de la terre; il rend le noir éblouissant et il redonne à sa terre natale la dimension historique et mythique que deux cents ans de collaboration et de colonisation ont tenté de rogner. enfin, surtout, îl montre l'aventure toujours possible, à recommencer, cette aventure vers laquelle allaient les pères avant qu'elle ne devienne empêchée:

mais donne la main à toutes les rencontres, pays

ô toi qui apparais

par tous les chemins défoncés de ton histoire

aux hommes debout dans l'horizon de la justice

qui te saluent

salut à toi territoire de ma poésie

salut les hommes des pères de l'aventure

(Compagnon des Amériques) 
Homme d'une anthropoésie comme il l'appelle, d'une poésie qui est une aventure de l'anthropologie, ses textes sont souvent des fragments, sont souvent en devenir, tout comme sa terre l'est, et le destin des hommes auquel il se relie.

1. L'article qui suit reprend dans une grande mesure les conclusions de ma thèse de 3e cycle, intitulée «La poétique de Gaston Miron». L'accent est donc mis sur les résultats auxquels a mené l'étude du rythme de la poésie de Miron davantage que sur l'analyse elle-même, encore que des exemples soient donnés qui actualisent la démarche théorique utilisée.

2. Plus particulièrement Critique du rythme, Paris, Verdier, 1982, 713 pages.

3. Émile Benveniste, "La notion de 'rythme' dans son expression linguistique", in Problèmes de linguistique générale 1, Paris, Gallimard, Tel, 1966, pp. 327-335

4. Ezra Pound, Literary Essays, London, Faber, 1974, p. 9: «Je crois en un'rythme absolu' un rythme qui est, en poésie ce qui correspond exactement à l'émotion ou à la nuance de l'émotion exprimée. Le rythme d'un homme doit être interprétatif, il sera, donc, à la fin, le sien, non imitatif, non imitable».

5. Émile Benveniste, op. cit., p. 336.

6. Je renvoie en particulier à MOLINO, Jeañ et TAMINE, Joëlle, Introduction à l'analyse linguistique de la poésie, Paris, Presses Universitaires de France, 1982, 232 pages, et à LUSSON, Pierre, et ROUBAUD, Jacques, "Sur la devise de 'noeud et de feu' un sonnet d'Étienne Jodelle, essai de lecture rythmique», in Langue française, no 49, février 1981, pp. 49-67. La suprématie d'une soi-disant neutralité de l'analyse métrique des premiers est à rapprocher du parti-pris mathématique des seconds. $\mathrm{Ni}$ la métrique, ni les mathématiques ne touchent au sens; c'est là, apparemment, ce qui fonde leur «neutralité». Rien de moins neutre pourtant que le retour au dualisme du fond et de la forme que ces approches réactualisent et dans lesquelles on retrouve la nostalgie d'un académisme que le post-structuralisme ressuscite bruyamment, se trouvant théoriquement dans l'impasse que sa double censure du sujet et du message a engendrée.

7. Ezra Pound, op. cit., p. 352.

8 Émile Benveniste, Problèmes de linguistique générale 1, Paris, Gallimard, Tel, 1966, p. 51-52.

9. Henri Meschonnic, Pour la poétique II, Paris, Gallimard, 1973, p. 80.

10. Ivan Fonagy, "Les bases pulsionnelles de la phonation", in Revue française de psychanalyse, janvier 1966.

11. Gaston Miron, Courtepointes, Ottawa, Publication du département des lettres françaises, Éditions de l'Université d'Ottawa, 1975, 57 pages.

12. MIRON, Gaston, l'Homme rapaillé, Montréal, Presses de l'Université de Montréal, 1970, 171 pages.

13. MIRON, Gaston, op. cit., p. 122-130.

14. Gaston Miron, «Femme sans fin», in Possibles, vol. 4, no 3-4, 1980, pp. 272-284.

15. Essais sur le Québec contemporain (La contribution d'Albert Faucher et de Maurice Lamontagne), Québec, 1953, p. 28, cité par SAINT-AUBIN, Bernard, Duplessis et son époque, Ottawa, Editions La Presse, 1979, p. 71.

16. Ibid.

17. Emile Benveniste, «La nature des pronoms», in Problèmes de linguistique générale 1, Paris, Gallimard, Tel, 1966, P. 251.

18. Henri Menchonnic, op. cit., p. 259.

19. Ibid., p. 97. 